\title{
SEARCH FOR T-SHAPED QUANTUM WIRES IN CdTe/CdMg(Mn)Te SYSTEM
}

\author{
G. Cywiński, T. Wojtowicz, G. Karczewski, J. Kossut \\ Institute of Physics, Polish Academy of Sciences \\ Al. Lotników 32/46, 02-668 Warsaw, Poland \\ AND W. GEูBICKI \\ Institute of Physics, Warsaw University of Technology \\ Koszykowa 75, 00-661 Warsaw, Poland
}

\begin{abstract}
We attempted to produce and to investigate T-shaped wire structures of II-VI compounds. Our samples were grown on GaAs hybrid substrates in a two-stage growth process. The photoluminescence measurements resulted into two different possible polarization behaviors of recorder signal. We interpret one of these behaviors as due to quantum wire formation.
\end{abstract}

PACS numbers: 72.15.Rn, 73.20.Dx, 85.40.Ux

Among several alternative approaches to fabrication of quasi one-dimensional nanostructures (quantum wires - QWRs) from II-VI semiconductors, the in situ methods are quite attractive since they may result in structures with minimized surface damage by avoiding the etching stage involved in lithographic methods. The main goal of our work was to obtain and, later, to study properties of $T$-shaped quantum wires from $\mathrm{CdTe}$ and its ternary and/or quaternary compounds with $\mathrm{Mn}$ and $\mathrm{Mg}$. Such structures can be achieved in a two-stage molecular beam epitaxy (MBE) process consisting of a superlattice growth followed by an overgrowth of an additional quantum well on the cleaved edge of the original superlattice (Fig. 1). The advantage of the method is related to a possibility of width control of each constituent layer with a high precision characteristic of MBE. The idea of the method was put forward by Yia-Chung Chang et al. [1] and realized for the first time by Goni et al. [2] who have grown AlGaAs/GaAs T-shaped QWRs. Since then, there were several publications reporting on experimental work on T-shaped QWR in III-V compounds [3, 4]. In 1997 Wegscheider et al. reported the first fabrication of quantum dots by twofold cleaved edge overgrowth [5], which is a natural extension of original idea [1]. So far there are no reports on making T-shaped QWRs from II-VI compounds. This is related, partially, to the lack of suitable substrates and to the problem of internal strains.

We have attempted to avoid these problems by using hybrid substrates, i.e., by depositing on (001)-oriented GaAs ( 2 inch in diameter, $400 \mu \mathrm{m}$ or $100 \mu \mathrm{m}$ thickness) a specially designed sequence of thick buffer. The hybrid substrates used by 


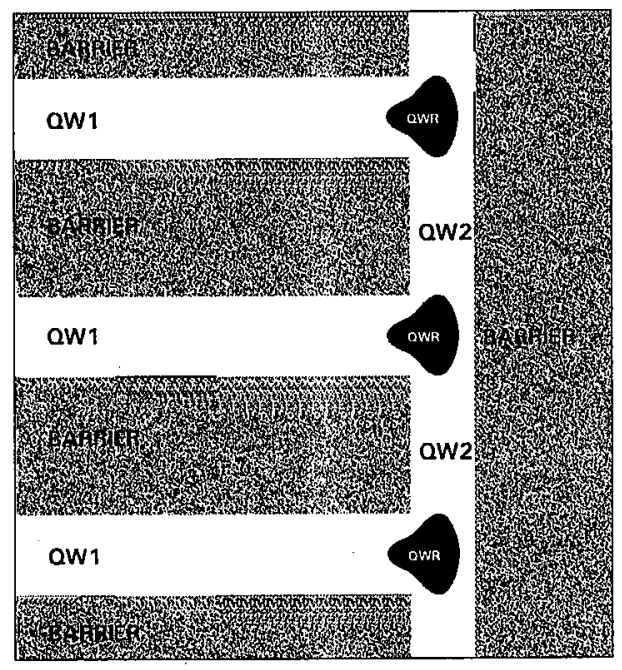

Fig. 1. Scheme of T-shaped wire structures. QW1 - quantum well in superlattice, QW2 - quantum well on cleaved (110) surface, QWR - T-shaped quantum wire structure, BARRIER1 - barrier in the first stage of growth, BARRIER2 - barrier in the second, overgrown stage. We have attempted to have the same composition in both barriers.

us were relatively thick to avoid problems related to heterointerfaces. More specifically GaAs substrates were covered by a thick $(4 \mu \mathrm{m}) \mathrm{CdTe}$ buffer, followed by a $2 \mu \mathrm{m} \mathrm{CdMnTe}$ barrier layer (or, optionally, CdMgTe or CdMgMnTe), followed only then by a superlattice ( 16 or 20 periods). The structure was further capped with a $2 \mu \mathrm{m}$ layer composed of the ternary or quaternary material previously used as the buffer underneath the superlattice. The superlattice consisted of either 10 or 12 monolayers (ML) wide quantum wells separated by 100 ML wide barriers. Such wafers were removed from the MBE chamber, had the GaAs substrate thinned down to approximately $100 \mu \mathrm{m}$ (in the case when $400 \mu \mathrm{m}$ thick GaAs substrates were used) and then cleaved into rectangular samples. The samples mounted in special holders were reinserted into the MBE growth chamber and cleaved in vacuum, thus exposing their (110) edge. Next, we deposited a single $12 \mathrm{ML}$ wide quantum well, and 160 ML thick barrier on the cleaved (110) edge. Earlier we optimized growth conditions on (110)-oriented substrates and we found that growing on cleaved hybrid substrates permitted to obtain sharp photoluminescence signals due to recombination of excitons from (110)-oriented quantum wells [6].

Unfortunately, not every overgrowth process resulted in a good quality QW displaying narrow photoluminescence (PL) lines, probably due to a bad quality of (110)-cleaved surface.

Photoluminescence (excited by $514.5 \mathrm{~nm}$ argon laser line) was studied at $4 \mathrm{~K}$ in the system equipped with a microscope. The exciting beam was focused to form $10 \mu \mathrm{m}$ diameter spot on the overgrown surface. The PL signal was collected by the same microscope objective system and after passing through the analyzing polarizer was dispersed in a double monochromator with the CCD camera. 
The signal and laser beams were almost entirely enclosed inside the monochromator and an aligning box, thus reducing the possibilities of studies of polarization properties and absolute signal intensity. However, this setup allows us to obtain the PL spectra in three cases: (i) without any analyzing polarizer, (ii) with a linear polarization analyzing plate either parallel to the $\mathrm{T}$-wire axis $(X Y$-configuration) or (iii) perpendicular to this axis ( $Y Y$-configuration).

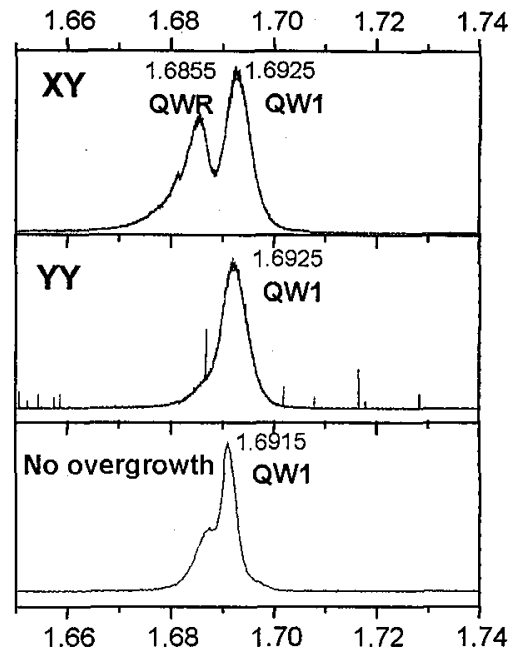

(a)

Energy $(\mathrm{eV})$



Fig. 2. Photoluminescence spectrum of overgrowth structures. The polarization dependence of additional overgrowth peak has been interpreted as quantum wires existence. The left figure (a) is showing spectra of $\mathrm{CdTe}$ wire structure buried in $\mathrm{Cd}_{0.77} \mathrm{Mn}_{0.23} \mathrm{Te}$ barriers (sample 110795A) and the right figure the wire structure $\mathrm{Cd}_{0.97} \mathrm{Mn}_{0.03}$ Te buried in barriers $\mathrm{Cd}_{0.72} \mathrm{Mg}_{0.25} \mathrm{Mn}_{0.03} \mathrm{Te}$ (sample 070497A).

In Fig. 2 we show the PL spectra of two samples containing the T-shaped wire structures. Figure 2a shows PL maxima from the sample, consisting of CdTe QWs embedded between $\mathrm{Cd}_{0.77} \mathrm{Mn}_{0.23}$ Te barriers (QW1 and QW2 are $12 \mathrm{ML}$ thick CdTe layers). The uppermost spectrum shows the PL signal from the overgrown region in the parallel orientation of the polarizer ( $X Y$-configuration). We can observe two peaks (at $1.6855 \mathrm{eV}$ and at $1.6925 \mathrm{eV}$ ). The lowest spectrum in this figure shows the PL signal from the same cleaved superlattice (SL) without overgrown layers on it. Therefore, it is obvious that the peak at $1.6925 \mathrm{eV}$ is due to emission from QW1. The peak at $1.6855 \mathrm{eV}$ (clearly visible in the uppermost spectrum) is, therefore, definitely related to the overgrown structure. The middle spectrum in Fig. 2a shows the PL signal collected from the same point of the overgrown structure, but in the perpendicular polarization $(Y Y)$. Only one peak is visible in this spectrum. We see, then, that the PL feature introduced by the overgrown layer is very anisotropic as could be expected for the case of quantum wire. We, 
therefore, would like to tentatively associate this features to the emission from a T-shaped $1 \mathrm{D}$ quantum wire.

The analogous situation occurs in Fig. 2b, where we present PL spectra from the sample containing the $\mathrm{T}$-shaped wire structure made of $\mathrm{Cd}_{0.97} \mathrm{Mn}_{0.03} \mathrm{Te}$ QWs (both QW1 and QW2 are $12 \mathrm{ML} \mathrm{Cd}_{0.97} \mathrm{Mn}_{0.03} \mathrm{Te}$ layer) embedded in $\mathrm{Cd}_{0.72} \mathrm{Mg}_{0.25} \mathrm{Mn}_{0.03} \mathrm{Te}$ barriers. Two upper spectra in Fig. $2 \mathrm{~b}$ are in close analogy to the spectra in Fig. 2a. However, we observe the peak related to PL from the overgrown edge structure in both polarizations. Therefore, we associate the weak feature visible in the $Y Y$-configuration at the highest energy as due to the emission from quasi 2D QW1.

Again, the emission that is most highly anisotropic (and completely missing in $Y Y$-configuration) - occurring at the lowest energy in the uppermost spectrum in Fig. $2 b$ - can be tentatively ascribed to 1D QWR.

A different behavior was observed in the case of yet another sample, which was produced by the cleaved edge overgrowth of $10 \mathrm{ML} \mathrm{CdTe} / 100 \mathrm{ML}$ $\mathrm{Cd}_{0.56} \mathrm{Mg}_{0.44} \mathrm{Te}$ supperlattices by $12 \mathrm{ML}$ CdTe well followed by $\mathrm{Cd}_{0.67} \mathrm{Mg}_{0.33} \mathrm{Te}$ barrier layer. The spectra from this sample showed also an additional peak related to the overgrown structure, however it was not anisotropic at all. This peak was, surprisingly, quite strong. Its origin can be explained in the following way: the energy of the grand level in QW2 is much smaller than in QW1. Consequently the excitation of QW1 is very effectively transferred enhancing the excitation into QW2. Additionally, QW2 is most probably less perfect, and thus, excitons in it are more localized and recombine radiatively very efficiently.

Therefore, it is most likely that the additional peak in this sample is related to the enhanced PL from the QW2 signal and is not related to the quasi one-dimensional T-shaped QWRs.

In conclusion, we have observed additional PL peaks that can be interpreted as related to the $\mathrm{PL}$ signal from $\mathrm{T}$-shaped wire, however further experiments are required to unambiguously confirm this interpretation.

This work was partially supported by the Committee for Scientific Research (Poland) under grant PBZ028-11 and by Volkswagen Foundation.

\section{References}

[1] Yia-Chung Chang, L.L. Chang, L. Esaki, Appl. Phys. Lett. 47, 1324 (1985).

[2] A.R. Goni, L.N. Pfeiffer, K.W. West, A. Pinczuk, H.U. Baranger, H.L. Stormer, Appl. Phys. Lett. 61, 1956 (1992).

[3] H. Akiyama, T. Someya, H. Sakaki, Phys. Rev. B 53, R4229 (1996).

[4] M. Yoshita, H. Akiyama, T. Someya, H. Sakaki, J. Appl. Lett. 83, 3777 (1998).

[5] W. Wegscheider, G. Schuedelberck, G. Abstreiter, M. Rother, B. Bichler, Phys. Rev. Lett. 70, 1917 (1997).

[6] G. Cywiński, T. Wojtowicz, K. Kopalko, G. Karczewski, J. Kossut, Acta Phys. Pol. A 94, 281 (1998). 